\title{
The Effect of EDTA on the Nucleation Kinetics and Mechanical Properties of KDP Crystal
}

\author{
A. Rahman ${ }^{a}$ and J. Podder ${ }^{b^{*}}$ \\ ${ }^{\mathrm{a}}$ Department of Basic Sciences and Humanities (Physics), University of Asia Pacific, Dhaka-1209, \\ Bangladesh \\ ${ }^{b}$ Department of Physics, Bangladesh University of Engineering and Technology, Dhaka-1000, \\ Bangladesh
}

Received 9 January 2012, accepted in final revised form 24 May 2012

\begin{abstract}
Potassium di-hydrogen phosphate (KDP) has been successfully grown in pure form and doped with ethylene diamine tetra acetic acid (EDTA) by low temperature solution growth technique and nucleation kinetics of KDP crystals in doped and undoped solutions were measured and analyzed. The induction period $\tau$ was measured and experiments were performed at selected degrees of supersaturation and the critical nucleation parameters like energy of formation of the critical nucleus $\left(\Delta G^{*}\right)$, radius of the critical nucleus $r^{*}$, nucleation rate $J$, number of the molecules in the critical nucleus $i^{*}$ have been calculated based on the classical theory of nucleation. The grown crystals have been subjected to study the mechanical property. The microhardness test was carried out on (100) plane. The load dependent hardness and work hardening coefficient was measured.
\end{abstract}

Keywords: EDTA; KDP crystal; Induction period; Hardness; Work hardening coefficient.

@ 2012 JSR Publications. ISSN: 2070-0237 (Print); 2070-0245 (Online). All rights reserved.

doi: http://dx.doi.org/10.3329/jsr.v4i3.9415 J. Sci. Res. 4 (3), 533-540 (2012)

\section{Introduction}

A comprehensive theory of nucleation from solution does not exist at present but there is growing evidence that it is a two-step process: the formation of liquid like clusters of solute molecules followed by the rate-limiting organization of such a cluster into protocrystals [1]. Nucleation, the initial step in the process of crystallization involving the formation of a critical nucleus, is still poorly understood [2]. In crystal growth, solutions are classified into three different categories: undersaturated solution, saturated solution and supersaturated solution. In undersaturated and saturated solutions crystal growth is not possible. Crystallization from solution can occur only in supersaturated solutions. Supersaturation can be achieved in several ways - for example by cooling method, by

\footnotetext{
* Corresponding author: jpodder@phy.buet.ac.bd
} 
evaporation method and by changing the $\mathrm{pH}$ of the solution. Supersaturated solution properties are important in the development of fundamental understanding of nucleation and crystal growth mechanisms. At any time supersaturated solution, although in thermal equilibrium, is not at thermodynamic equilibrium. At all times, the supersaturated solution is a mixture of dimers, trimers, tetramers etc. Nucleation occurs when clusters reach a critical size where the Gibbs free energy changes for growth of the clusters is negative. Eventually, a critical size is reached and a crystal is born [3].

The growth of KDP crystals in larger dimensions than other non-linear crystals [4] has made it to be used in a wide range of applications. The growth technique for production of commercial crystals is based on gradual temperature reduction. So attention is given to grow crystals from solutions with faster rates by adopting faster cooling rates. Srinivasan et al. [5] introduced a novel method to enhance the metastable zone width for crystal growth from solutions by adding the chelating agent EDTA. A large number of papers were devoted to the growth kinetic analysis of prismatic and pyramidal faces of KDP [6-10]. The influence of impurities on the growth kinetic of KDP was also presented [11-13].

KDP belongs to the scalenohedral (twelve sided polyhedron) class of tetragonal crystal system. It has a tetramolecular unit cell having the dimensions given as $a=b=$ $7.448 \AA$ and $c=6.977 \AA$. KDP is ferroelectric well below room temperature and the curie temperature $\mathrm{T}$ is $123 \mathrm{~K}$. KDP is soluble in water and the molecular weight is 136.09 . With the aim of improving the quality of KDP crystals with better mechanical properties, an attempt has been made in the present work to grow the KDP crystals by doping it with EDTA (concentration of $0.5 \mathrm{~mol} \%$ ) and study the effect on the nucleation parameters and mechanical properties.

\section{Experiment}

\subsection{Determination of induction period}

Induction period ' $\tau$ 'can give information about the mechanism of solid phase formation and the process of growth from critical nuclei to detectable crystals [14-15] and is determined experimentally for KDP solutions with and without the presence of EDTA by adopting isothermal method [16]. The supersaturated solutions were prepared to conduct induction time experiments. The required level of supersaturation was achieved by dissolving the required amount of KDP (with and without EDTA) in the solvent, which is above the saturation concentration. The mixture is then heated to a few degrees above the saturation temperature $\left(30^{\circ} \mathrm{C}\right)$ to ensure that all the material is dissolved. The solution is then cooled to the saturation temperature $\left(30^{\circ} \mathrm{C}\right)$ where the solution becomes supersaturated to the required degree of supersaturation. Once the nucleation occurred, it grew quickly and a bright sparkling particle was seen. The time of observation of the sparkling particle in the cell from the time at which the solution reaches the saturation temperature gives the induction period of nucleation. Experiments were performed at 
selected degrees of supersaturation $\left(C / C^{*}\right)$, viz., 1.07, 1.1, 1.13, 1.16, $(C$ being the concentration of solute in supersaturated solution and $C^{*}$ is the saturated concentration).

\subsection{Nucleation kinetics of KDP and doped KDP}

The relationship between rate of nucleation (representing the number of nuclei formed per unit time per volume) and induction period can be expressed as [17]

$$
\begin{aligned}
& J=\frac{1}{\tau}=A \exp \left(-\frac{\Delta G^{*}}{k T}\right)=A \exp \left(-\frac{16 \pi \sigma^{3} v^{2}}{3 k^{3} T^{3}(\ln S)^{2}}\right) \\
& \ln (\tau)=-\ln (A)+\frac{16 \pi \sigma^{3} v^{2}}{3 k^{3} T^{3}(\ln S)^{2}}
\end{aligned}
$$

where $\tau$ is the induction period of the solution at temperature $T, v$ is the molar crystal volume, $k$ is the Boltzmann constant and $A$ is constant. $S$ is the supersaturation ratio $(S=$ $\left.C / C^{*}\right)$. The function, $\ln A$ weakly depends on temperature and hence there is a linear dependence between $\ln \tau$ and $1 /(\ln S)^{2}$ at constant temperature. A plot of $\ln \tau$ against $1 /(\ln$ $S)^{2}$ is a straight line [11]. The intercept of the straight line on the y-axis gives the value of $\ln A$. Eq. (3) suggests a straight line fit for $\ln \tau$ against $1 /(\ln S)^{2}$ with a slope $m$ given in ref. [18]:

$$
m=\frac{16 \pi \sigma^{3} V^{2} N_{A}}{3 R^{3} T^{3}}
$$

In some studies, linear relationship between $1 /(\ln S)^{2}$ and $\ln (\tau)$ is not represented by one line, but two different straight lines can be drawn, one of which represents homogeneous nucleation and the other represents heterogeneous nucleation. The interfacial energy $\sigma$ of the solid relative to the solution has been calculated from the slope of the line as

$$
\sigma^{3}=\frac{3 R^{3} T^{3} m}{16 \pi V^{2} N_{A}}
$$

where $V$ is the specific volume and is obtained by $V=\frac{\text { Molecular weight }}{\text { Density } \times N_{\mathrm{A}}}, R$ is the gas constant and $N_{A}$ is the Avogadro's number. The energy of formation of a critical nucleus has been evaluated from the experimental data as

$$
\Delta G^{*}=\frac{R T m}{(\ln S)^{2}}
$$

The radius of the nucleus in equilibrium with its solution has been computed as [19]

$$
r^{*}=\frac{2 \sigma V}{k T \ln S}
$$


The number of molecules in the critical nucleus is expressed as [20]

$$
i^{*}=\frac{4 \pi\left(r^{*}\right)^{3}}{3 V}
$$

\subsection{Characterization studies}

Hardness test: Microhardness of a crystal is its capacity to resist indentation. The hardness of a material depends on different parameters such as lattice energy, Debye temperature, heat of formation and interatomic spacing [21]. To evaluate the Vickers hardness number, an indentation time of $10 \mathrm{sec}$ was applied on the (100) face of the crystal for loads 25 to $200 \mathrm{~g}$. The distance between any two indentations was maintained to be greater than five times the diagonal length in order to avoid any mutual influence of the indentations. Maximum indentor load applied was $200 \mathrm{~g}$, above this load microcracks were observed around the impression and hence readings were not taken for higher loads. The Vickers microhardness number was calculated using the expression

$$
H_{V}=1.854\left(\frac{p}{d^{2}}\right) \frac{k g}{m m^{2}}
$$

here $H_{V}$ is the Vickers hardness number in $\mathrm{kg} / \mathrm{mm}^{2}, p$ is the applied load in $\mathrm{kg}$ and $d$ is the average diagonal length of the indentation in mm. Fig. 1 shows load vs. Vickers hardness number for pure and EDTA doped KDP crystal. At lower load, there is an increase in the hardness with load, which can be attributed to the work hardening of the surface layers.

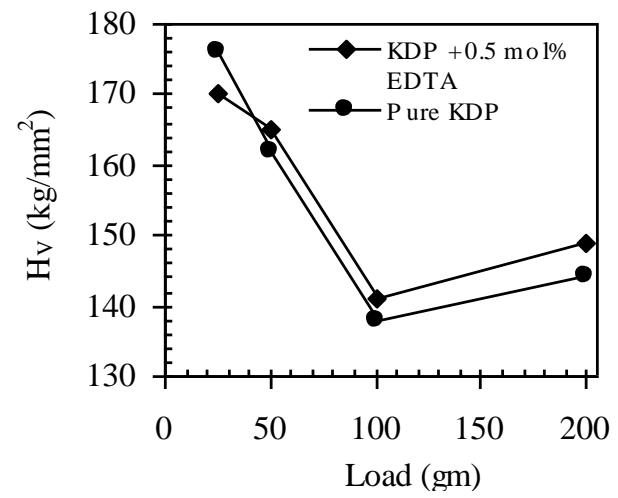

Fig. 1. $H_{\mathrm{V}} v s$. load for pure and doped KDP crystal.

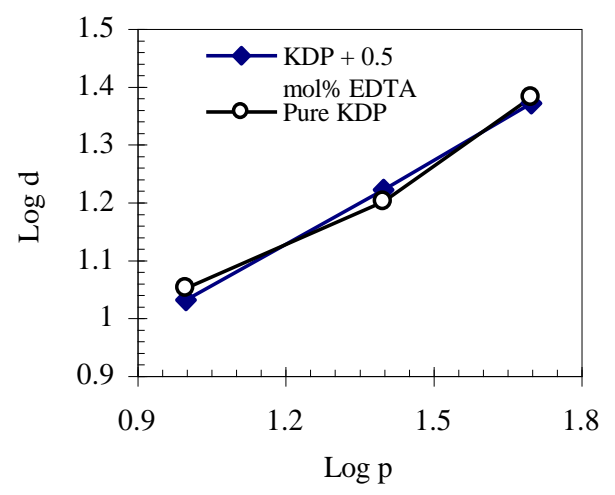

Fig.2. $\log p$ vs. $\log d$ for pure and doped KDP crystal.

Work hardening coefficient $n$, a measure of the strength of the crystal, is computed from the $\log p-\log d$ plot. The plot of $\log p-\log d$ (Fig. 2) yields a straight line and work hardening index or Meyer index, $n$ is obtained from the slope of the line. According to Meyer's law [22],

$$
p=k_{1} d^{\mathrm{n}}
$$


The hardness value and work hardening coefficient are found to increase, which proves that EDTA added KDP crystal has higher mechanical strength.

\section{Results and Discussion}

The induction period is measured and the critical nucleation parameters like interfacial energy $\sigma$, energy of formation of the critical nucleus $\Delta G^{*}$, radius of the critical nucleus $r^{*}$, nucleation rate $J$, number of the molecules in the critical nucleus $i *$ have been calculated and shown in Figs. 3 to 7 . Investigations show that due to incorporation of EDTA, the induction period $\tau$ increases from 181 to $1394 \mathrm{~s}$ for KDP for supersaturation ratio $S=1.07$ (Fig. 8). The energy of formation of the critical nucleus $\Delta G^{*}$ increases from 1.93 to 3.54 $\mathrm{kJ}$ for KDP for supersaturation ratio $S=1.07$.

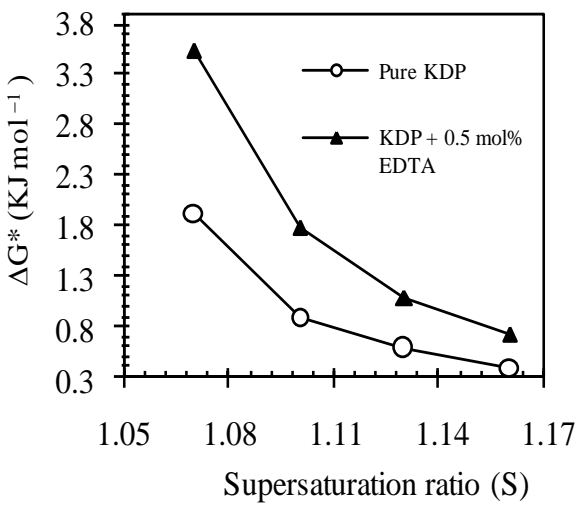

Fig. 3. Energy $\Delta G^{*}$ vs. supersaturation ratio of pure KDP and EDTA added KDP solution.

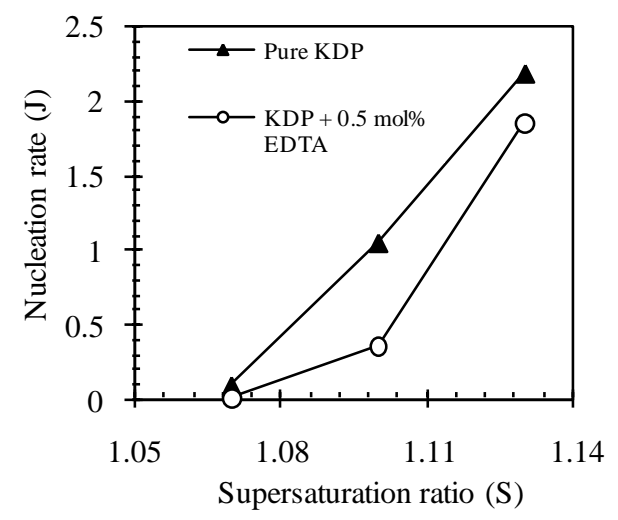

Fig. 5. Nucleation rate $(J)$ vs. supersaturation ratio of pure KDP and EDTA added KDP solution.

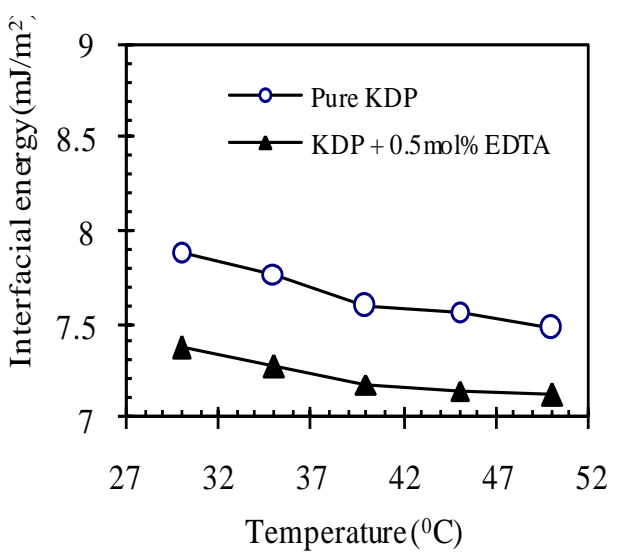

Fig. 4. Interfacial energy $v s$. temperature of pure KDP and EDTA added KDP.

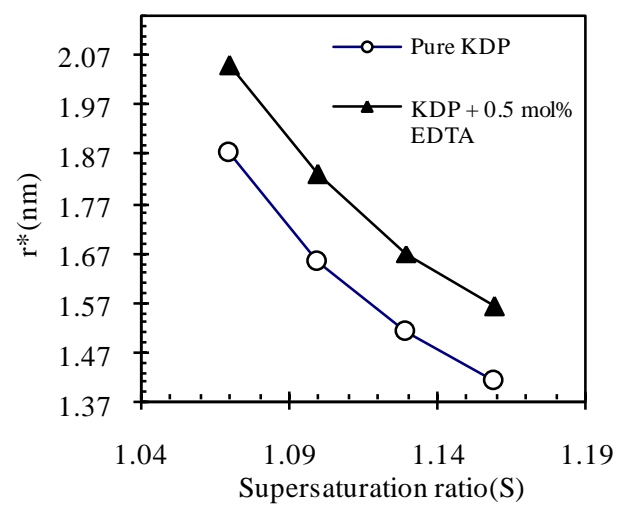

Fig. 6. Radius of critical nucleus vs. supersaturation ratio of pure KDP and EDTA added KDP solution. 


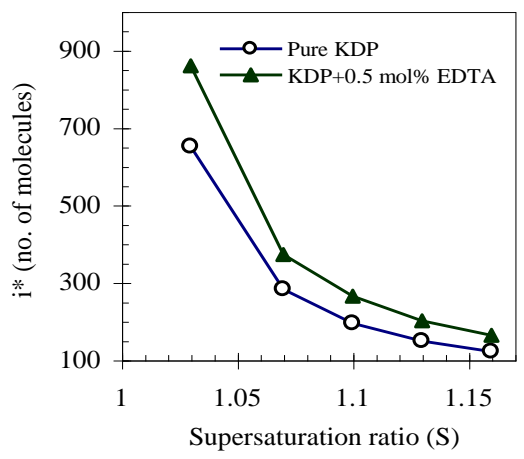

Fig. 7. Number of molecules in the critical nucleus $(i *)$ vs. supersaturation ratio of pure KDP and EDTA dded KDP solution.

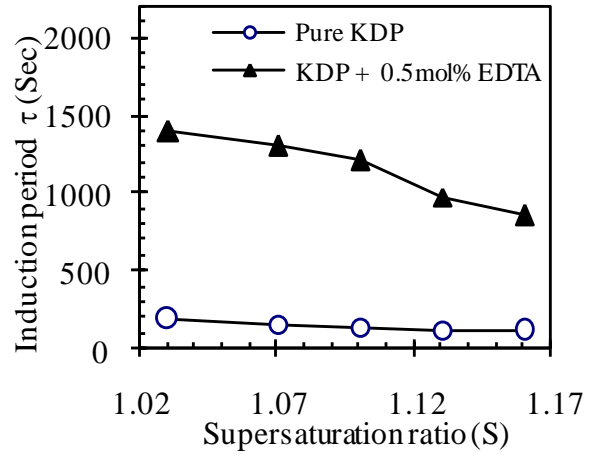

Fig. 8. Induction period vs. supersaturation ratio of pure KDP and EDTA added KDP solution.

The radius of the critical nucleus $r^{*}$ increases from 1.87 to $2.05 \mathrm{~nm}$ for KDP for supersaturation ratio $S=1.07$. The number of molecules in the critical nucleus $i *$ increases from 282 to 372 for KDP for supersaturation ratio $S=1.07$. The nucleation rate $J$ decreases from $0.09 \times 10^{24}$ to $0.0053 \times 10^{24}$ nuclei/ $/ \mathrm{cm}^{3}$.s for KDP for supersaturation ratio $S$ $=1.07$.

Table 1.

\begin{tabular}{cc}
\hline Material & $\begin{array}{c}\text { Work hardening } \\
\text { coefficient } n\end{array}$ \\
\hline Pure KDP & 1.67 \\
KDP +0.5 mol\% EDTA & 2.1 \\
\hline
\end{tabular}

From Vickers micro-hardness, the work hardening coefficient ' $n$ ' has been determined which is observed to increase from 1.67 to 2.1 (Table 1) with the addition of EDTA (concentration of $0.5 \mathrm{~mol} \%$ ). This can be attributed to the fact that the crystals when doped with EDTA, the metal ions which were acting as defects in the crystal are being removed from the solution. So the bonds become stronger among the KDP ions and they are having relatively more mechanical strength. Fig. 9 shows the photograph of the as grown crystal of $0.5 \mathrm{~mol} \%$ EDTA added KDP.

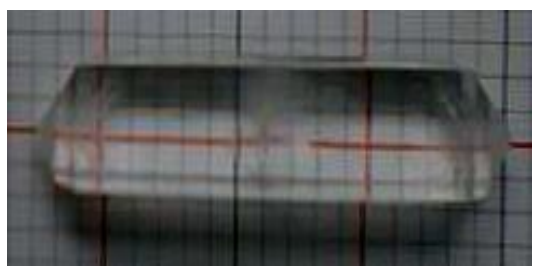

Fig. 9. Photograph of the as grown crystal of $0.5 \mathrm{~mol} \%$ EDTA added KDP. 


\section{Conclusions}

Pure and EDTA added KDP crystals were grown by slow evaporation technique. From the study of induction period, the critical nucleation parameters are calculated and observed that the values of $\tau, \Delta G^{*}$ increases with the addition of EDTA. It is observed that the values of $\tau, \Delta G^{*}, r^{*}, i^{*}$ increases but $J$ decreases with the addition of EDTA. It was noted that with the increase in super saturation, the free energy change for the formation of a critical nucleus $\left(\Delta G^{*}\right)$ decreases with radius $\left(r^{*}\right)$. This favors the easy formation of nucleation in solutions at higher supersaturations. In this work, it is observed that the incorporation of EDTA is increasing the critical size of the nucleus. So that the probability of nucleation is decreasing considerably and this is why, the number of the formation of tiny seed crystals are observed to decrease i.e. nucleation rate $J$ decreases. The microhardness measurement studies illustrate an increase in hardness and work hardening coefficient with the addition of EDTA. This study may prove to be helpful to obtain high quality single crystals as well as to apply for faster growth rates of KDP crystals.

\section{Acknowledgement}

The authors are thankful to Dr. A. Gaffur, Senior Engineer, BCSIR laboratory, Dhaka for his cooperation to use the microhardness instrument in his laboratory.

\section{References}

1. J. D. Shore, D. Perchak, and Y. Shnidman, J. Chem. Phys. 113, 6276 (2000). http://dx.doi.org/10.1063/1.1308517

2. D. W. Oxtoby, Acc. Chem. Res. 31, 91 (1998). http://dx.doi.org/10.1021/ar9702278

3. G. Anandha Babu and P. Ramasamy, Cryst. Res. Technol. 43, 626 (2008). http://dx.doi.org/10.1002/crat.200711076

4. N. P. Zaitseva, J. Atheron, R. Rozsa, L. Carman, I. Smolsky, M. Runkel, R. Ryon, L. James, J. Cryst. Growth 197, 911-920 (1999). http://dx.doi.org/10.1016/S0022-0248(98)01095-1

5. K Srinivasan, K Meera and P ramasamy, J. Cryst. Growth 205, 457 (1999)

6. A. A. Chernov, L. N. Rashkovich, J. Cryst. Growth 84, 389 (1987). http://dx.doi.org/10.1016/0022-0248(87)90265-X

7. A. A. Chernov, Z. Phys. Chemie, Leipzig 269, 941 (1988).

8. A. A. Chernov, Contemporary Physics 30, 251 (1989). http://dx.doi.org/10.1080/00107518908225517

9. H. V. Alexandru, J. Cryst. Growth 205, 215 (1999). http://dx.doi.org/10.1016/S0022-0248(99)00246-8

10. H. V. Alexandru, C. Berbecaru, Al. Grancea, V. Iov, J. Cryst. Growth 166, 162 (1996). http://dx.doi.org/10.1016/0022-0248(96)00037-1

11. A. Rahman and J. Podder, Int. J. Optics 2010, Article ID 978763, 5 pages. http://dx.doi.org/10.1155/2010/978763

12. J. Podder, J. Cryst. Growth, 237-239, 70 (2002).

13. J. Podder, S. Ramalingom and S. N. Kalkura, Cryst. Res. Technol. 36, 551 (2001) http://dx.doi.10.1002/1521-4079(200107)36:6<549:AID-CRAT549>3.0.Co;2-7.

14. D. Ackermann, P. Brown and S. R. Khan, Urol. Res. 17, 147 (1989).

15. O. Sohnel, and J. W. Mullin, J. Colloid and Interface Sci., 123, 43 (1988). 
16. N. P.Zaitseva, L. N. Rashkovich, and S. V. Bogatyreva, J. Cryst. Growth. 148, 276 (1995). http://dx.doi.org/10.1016/0022-0248(94)00606-7

17. O. Sahin, H. Dolas, and H. Demir, Cryst. Res. Technol. 42, 766 (2007). http://dx.doi.org/10.1002/crat.200710904

18. S. Arjunan, R. Mohan Kumar, R. Mohan and R. Jayavel, Cryst. Res. Technol. 43, 417 (2008). http://dx.doi.org/10.1002/crat.200711036

19. T. Kanagasekaran, M. Gunasekaran, P. Srinivasan, D. Jayaraman, R. Gopalakrishnan, and P. Ramasamy, Cryst. Res. Technol. 40, 1128 (2005). http://dx.doi.org/10.1002/crat.200410504

20. R. Sankar, C. M. Raghavan, and R. Jayavel, Cryst. Res. Technol. 41, 919 (2006). http://dx.doi.org/10.1002/crat.200510696

21. C. Vesta, R. Utharkumar, C. J. Raj, A. J. Varjula, J. M. Linet, and S. J. Das, J. Mater. Sci. Technol. 23 (6), 855 (2007).

22. A. Rahman and J. Podder, Indian J. Phys. 86 (1), 15 (2012). http://dx.doi.org/ 10.1007/s12648-012-0003-8 